\title{
Model of Mechanical Interaction of Mesenchyme and Epithelium in Living Tissues
}

\author{
Jiř́i Kroc ${ }^{\star \star}$ \\ Department of Mechanics, University of West Bohemia in Pilsen \\ Univerzitni 22, 30614 Pilsen, Czech Republic \\ kroc@c-mail.cz \\ http://www.c-mail.cz/kroc
}

\begin{abstract}
Developmental biology describes how tissues, organs, and bodies are made from living cells. There exists a large body of biological data about developmental processes but there is still not ultimate understanding of how the whole orchestra of all involved processes is working. It is the place where mathematical modelling could help to create biologically relevant models of morphological development. The morphological development could be mathematically decomposed into three distinct but mutually interconnected parts, namely to mechanical response of tissues, signalling by chemicals, and switching of cells into different types by a gene regulatory network. This paper is focussed to the part dealing with mechanical interaction of growing mesenchyme and epithelium within a living tissue modelled by a set of nodes interconnected by deformable bars as in tensegrity models.
\end{abstract}

\section{Introduction}

Developmental biology is describing - simply said - how the whole body of a living creature could be created from a single cell. It is a very vital field that produce a lot of genetic, signalling and morphological data - but current knowledge does not cover the whole field so far. New, clever experimental approaches bring a constant flux of data. The most important point is that there is not unique understanding of how those single parts are working together. From a certain distance and using a kind of metaphor, we could say that we roughly know how every single player perform its part but we do not understand how the whole orchestra is working. This is the point where mathematical models take their part. They enable us to build adequate models of developmental biology using biologically relevant input data where outputs could be directly compared to another biologically observed data.

From the mathematical point of view, the whole problem could be easily decomposed into three distinct parts. Namely, evolution of morphology-i.e., topology in the mathematical sense - by growth, signalling by specific chemicals

\footnotetext{
** This work was supported by the Czech Ministry of Education, Youth and Sports under grant number MSM 4977751303.
} 
produced by different types of cells, and actions of gene regulatory network which tells every single cell if it has to undergo - in the specific morphological and chemical context - a cell type change or not.

The whole problem becomes quite complicated not only due to size of growing morphology but, as well, due to size of gene regulatory network itself. Therefore, there is a good reason to start with a much simpler case that will capture the essence of this type of modelling. The natural beginning is to model growth of a tooth. From biological point of view, it is well known that most of - if not all - interactions among all cells in developing tooth are encapsulated inside of growing tooth egg and its subsequent stages.

A growing tooth egg enter several developmental stages going from the egg, across cap, bell and to the final shape. It is well known that the crucial role in development of mammalian tooth-having crown with a relatively complex structure - is played by epithelial and mesenchyme growth which compete one with the other in speed of growth. Mesenchyme is encapsulated in epithelium, i.e. epithelium grows in two-dimensions and mesenchyme in three-dimensions. This leads to the situation that some parts of epithelium - which generates mechanical force - are imposed to higher strains. Places which encounter larger strains are transformed into so called knots - via gene regulatory network - which influence speed of growth of epithelium in surrounding and naturally leads to creation of crown by invagination of epithelium into mesenchyme.

Mathematical model of morphological development of one tooth will be built - and published in a series of papers - using complex systems where cellular automaton is employed as the mathematical tool expressing the complexity of the model through its parts [1,2, 3, i.e. growth by cell division, signalling to neighbouring cells by chemicals and transformation of cells into different cell types due to gene regulatory network. Concept of cellular automata enables a very detailed - spatially and in time - definition of behaviour of every part of the simulated topology. Some models of morphological development are already known but the problem is that they do not fully reflect biologically observed behaviour, e.g. some non-local computations of mechanical interactions are used what is not in coherence with biological observations. Those models typically use simplified gene regulatory networks, they do not work with correct mechanical interactions of cells, and use some other simplifications which might lead to improper biological outputs.

The general idea of the CA-model of mechanical behaviour of mesenchyme presented here comes from the tensegrity models [4, 5, 6, 7, 8] where the structure is composed from a set of two generic types of elements, one is under compression load (bars) and the other one under tensile load (strings). Spatial combination of those two types of elements leads to light and stable structures which are able to sustain large loads compared to classical structures. In the model, we work only with bars interconnecting nodes - originally located in the centre of each cellwhere one node belongs exactly to one cell. We employ knowledge achieved in structural design [9, 10, 11] regarded to elastic properties of structures to model mesenchyme but we know that behaviour of epithelium is richer then is used 
there. Gene regulatory network and diffusion of chemicals will be involved in the model later.

\section{Model}

The model of the mesenchymal and pseudo-epithelial interaction is built step by step. Firstly, one-dimensional model of mesenchyme and pseudo-epithelium is defined, studied, and carefully tested on tensile and compressible examples of metallic materials. Then a two-dimensional model is proposed with special attention to mesenchyme where mechanical properties of epithelium are simplified. It is fascinating how mechanical force could be created in living cells and tissues 12, 13, 14, 15. It is known that cells are working with small local actions leading to large global shape and force changes. We use ad hoc mechanisms leading to a very similar mechanical effects as in living tissues.

The model works with a network of nodes interconnected by bars. Each cell manage one node, see Figure 1. Bars are deformable by tension and compression. Strain is defined as

$$
\epsilon(t)=\frac{L(t)-L_{0}}{L_{0}},
$$

where $L_{0}$ is the initial length and $L(t)$ is its actual value at given time $t$. Use of this equation allows working with relative values used in the Hook's law.

The Hook's law represents linear dependence of force/stress on strain and is defined as

$$
\sigma(t)=\frac{F(t)}{A}=E \cdot \epsilon,
$$

where $\sigma(t)$ is stress $\left[\mathrm{N} / \mathrm{M}^{2}\right], F(t)$ is force $[\mathrm{N}]$, and $A$ is the cross-section of bars $\left[\mathrm{m}^{2}\right]$. This equation is elastic - i.e., linear - for the whole range of strain $\epsilon$ what is rather physically unrealistic because it allows to compress material to physically impossible strains.

Therefore, force $F$ is composed from two distinct parts. One is defined by Hooks law for tensile and compressible deformation which is taken from Equation 2 and the other one represents incompressibility of material. In our case, we expect that material could not be compressed below $\epsilon=-0.8$ of its original length $L_{0}$.

$$
\begin{aligned}
F & =E \cdot A \cdot \epsilon, & & \epsilon \in(-0.8,+\infty), \\
\epsilon & =-0.08, & & F<E \cdot A \cdot(-0.8) .
\end{aligned}
$$

In words, there is linear dependence of force $F$ on strain $\epsilon$ above $\epsilon=-0.8$, and the constant value of $\epsilon=-0.8$ is taken for force below the value of $E \cdot A \cdot(-0.8)$ due to incompressibility.

The reason why we use - as the first approximation - such dependence of force $F$ on strain $\epsilon$ is strictly defined by one well known physical constrain. It is known that compressibility of solids and liquids has a limit. The value of compression from the principle could not go bellow $\epsilon=-1$; it is even very difficult to approach 
values close to it. It stems from the law of mass conservation because mass could not be compressed to negative volumes.

To elucidate what happen if we take linear dependence of stress/force on deformation the following sequence of values of $L$ which is computed from $L_{0}$ by multiplication it with a factor of $2,1.1,0.9,0.5$, and 0.01 is inserted into Equation 1 and gives $\epsilon$ equal to $1,0.1,-0.1,-0.5$, and -0.99 , respectively. There is an obvious limit in compression equal to $\epsilon=-1$.

If force $F$ depends on strain $\epsilon$ linearly in the case of compression then we could not apply force lower then $(-E \cdot A)$. Values bellow are not physically relevant. Therefore, we have to use some kind of nonlinear dependence of force on deformation in region of compression as is done, for example, in Equation 3 .

The following set of three equations 4, 5, and 6 represents local equilibria for three types of cells used in the two-dimensional model, i.e. for top, bulk, and bottom - similarly for the left and right — cells

$$
\begin{array}{cl}
\sum_{(k, l) \in((-1,0),(0,-1),(1,0))} & F^{(k, l)}+F_{\text {surf }}^{\text {ext }}=0 \\
\sum_{(k, l) \in((-1,0),(0,-1),(1,0),(0,1))} & F^{(k, l)}+F_{\text {bulk }}^{\text {ext }}=0 \\
\sum_{(k, l) \in((1,0),(0,1),(-1,0))} & F^{(k, l)}+F^{f i x}=0
\end{array}
$$

where $F^{e x t}$ represents an externally applied force to the cell in our case and $F^{f i x}$ represents fixing force applied at the bottom, left, and right border cells. The last equation 6 represents the situation where cells are fixed during the whole simulation to the initially defined locations. Force $F^{f i x}$ balance the other forces, and therefore, no movement of those cells occurs. It could be simply done by keeping of all cell coordinates constant through the whole simulation.

In one-dimensional case - where a column of cells/bars is taken and the only allowed deformation works vertically_-,no equilibrium is computed at the bottom cell. The position is simply kept constant, i.e. the coordinates $x$ and $y$ are constant.

Vertical displacement of the top cell $y_{N}$ in the one-dimensional case is computed according to Equations 1, 2] and 4 (where $(k, 1)=\{(0,-1)\}$ ) and gives the following formula for tensile deformation

$$
y_{N}(t+1)=\frac{F^{e x t}}{E \cdot A} \cdot L_{0}+y_{N}(t)+L_{0}
$$

Vertical displacement of bulk cells in the one-dimensional case is computed from Equations 1, 2, and 5 (where $(\mathrm{k}, \mathrm{l})=\{(0,-1),(0,1)\})$-and after certain rearrangement - by mere averaging of positions

$$
y_{N}(t+1)=\frac{1}{2}\left(y_{N+1}(t)+y_{N-1}(t)\right)
$$

it could be done due to use of the linear Hooks law in compression. Please, note which simulation steps are taken on the left and right sides of the equation. Vertical displacement of all cells in the case of compression works with the same equations as in the case of tensile deformation. 


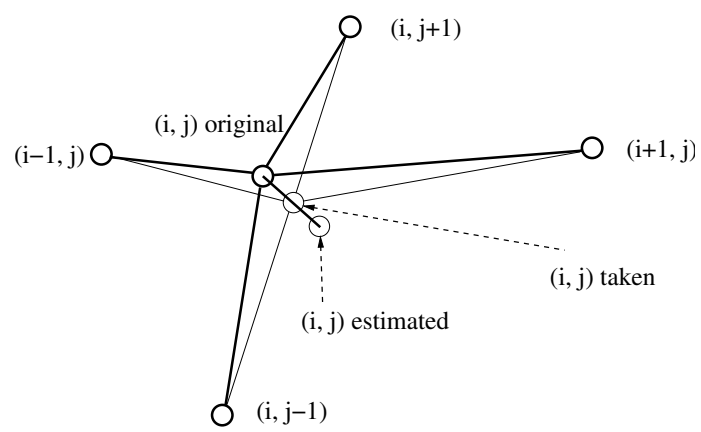

Fig. 1. The figure depicting the main idea of the used algorithm. The original position of the point managed by the updated cell $(i, j)$ is moved to the taken value according to values provided by four neighbouring cells which lays on the line connecting the original position with the estimated one.

Displacements in the two-dimensional case behaves in a much more complicated way then in the one-dimensional one. The main idea of the algorithm is explained in the Figure1 Whereas in the one-dimensional case the new position of the central cell could be simply computed by averaging positions of upper and lower cells, in the two-dimensional case we have to use an iteration method to find an estimated value.

In Figure 1 original position of the point managed by the updated cell $(i, j)$ is expected to be moved to the estimated position - given by solution of the equilibrium equations - which is the optimal solution of the problem regarded to data given by the neighbouring cells $(i-1, j),(i, j-1),(i+1, j)$, and $(i, j+1)$. If the point is moved to this estimated position then local change could be too fast and the updating algorithm looses its stability. Such instability could be removed when we take some value laying on the line interconnecting original and estimated value - called taken value. The distance from original point to the taken one is a predefined fraction $p$ from the distance of original and estimated points. Situation at the top cells is similar to the situation explained for the bulk cells except the fact that only three neighbouring cells are presented there, i.e. $(i-1, j),(i, j-1)$, and $(i+1, j)$.

Initially, the position of the cell under consideration is estimated using equations 4 5 and 6representing local equilibrium at the node. The algorithm used to find new position of nodes is working with halving of intervals in two dimensions. Then deformation limits are tested and the value called estimated achieved, i.e. compression could not go bellow $\epsilon=-0.8$. Finally, $p \%$ shift of the old position towards the estimated position of the cell under consideration is taken where $p$ is typically equal to $10 \%$ or $20 \%$.

\section{Results and Discussions}

Vertical displacement of the top cell $y_{i}$ was tested for several different external forces $F^{e x t}$ computed according to Equation 7 for tensile deformation and for 
compression in the one-dimensional case. It gives theoretically expected values. Testing of two-dimensional case was done for unloaded and loaded cases. Analysis of results is not as straightforward as in the one-dimensional case.

The following topology and data are used in the two-dimensional case - a block of $10 \times 10$ nodes which is anchored by cells laying at the left, bottom, and right edges of this block. The only cells allowed to move are those laying at the top edge of the block including all bulk cells. Cells number 5, 6, and 7 located at the top line of the block of cells - counted from left to right - are subjected to the external force $F=-2.5$ or -20 acting downwards, see Figure 2. Bars are having the Young modulus of $E=10^{5}$; the cross-section of them is $A=0.01 \cdot 0.01=10^{-4}$, the incompressibility threshold is set to 0.2 , and the initial distance of nodes $L_{0}=0.1$.

Snapshots depicting evolution of topology of a block of $10 \times 10$ nodes subjected to the force $F=-2.5$ or -20 which acts downwards at 5 th, 6 th, and 7 th cell at the top block of cells could be seen in Figures 2 and 3 . In the figures, nodes are depicted without bars. Several important observations were made upon this sequence. Firstly, symmetry breaking is present in the model because cells are moving to the left in the horizontal direction what is the most profound effect at the top cells. Secondly, as expected, the largest deformation is present at the place where external forces are applied. Thirdly, applied force squeeze the block and some top cells without applied external force are moved upwards for $F=-20$, e.g. third and fourth top nodes from the left. All those observations are coherent with experiments.

Model allows use of volume forces and it is possible to change the Young modulus locally from a place to a place but those properties of the model are not studied in this contribution. It is reserved for the future use of the model and for better description of behaviour of living tissues.

Solutions of the local equilibria equations [4, 5, and 6] have to be found by an iterative formula because it is the well known fact that there does not exist, in general, an analytical solution of a set of two or more nonlinear equations. It is found that presented algorithm fails when large simulation steps are used,
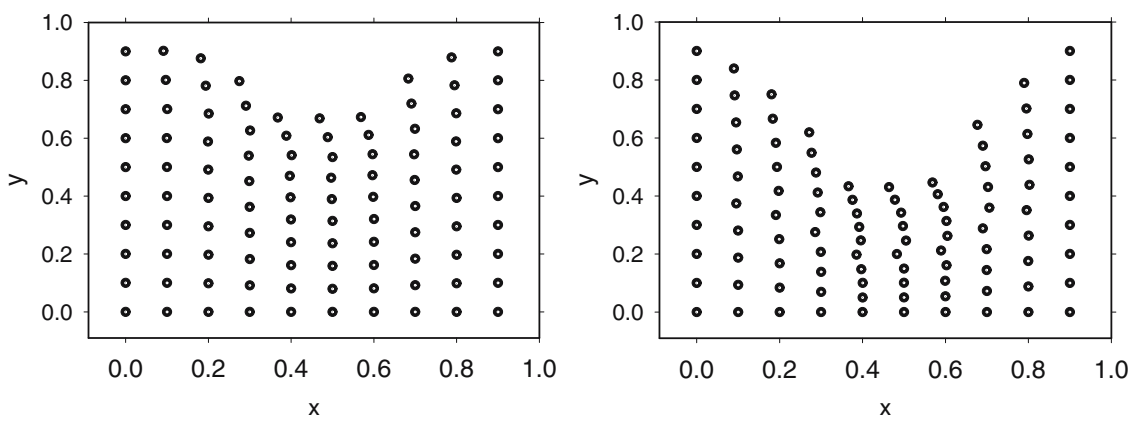

Fig. 2. Two configurations taken at simulation steps 100 and 300 - depicting evolution of topology of a block of $10 \times 10$ nodes stressed by force the $F=-20 \mathrm{~N}$ 


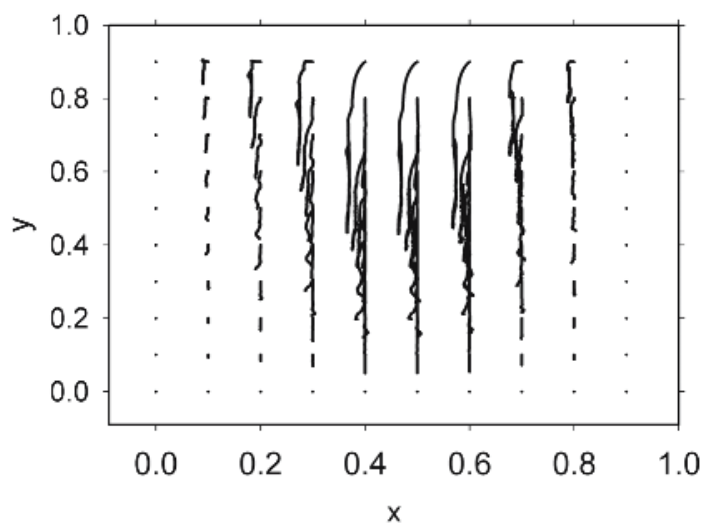

Fig. 3. A cumulative plot of all positions of all nodes for all simulated steps from 0 to 300 for the force $F=-20 \mathrm{~N}$ is shown here. Trajectories belonging to different nodes could be easily distinguished.

i.e. when the value of $p$ is approaching or equal to one. The reason is simple. Algorithm could exchange positions of neighbouring nodes for large steps, what is not physically acceptable. It automatically leads to instability of simulation and mixing of nodes. The proposed CA-model is working with physically relevant values as models using finite element method (FEM) do.

The topology used in this contribution represents the first, testing step towards a new model of tooth growth. The model describing behaviour of mesenchyme is defined here, i.e. mesenchyme is pressed by force from the top by a force that pseudo-epithelium generates, but - in general - mesenchyme could be pressed from any direction. In the future model, mechanical influence of epithelium would be taken into the game together with a gene regulatory network, and signalling chemicals produced according to this network which switch cells from one type into another one.

\section{Conclusions}

The first part-i.e. mechanical behaviour of mesenchyme and pseudoepithelium - of the model describing morphological development of tooth is proposed and tested in this contribution. In this model, mechanical influence of epithelium is mimicked by use of external force acting at the top side of square block of cellular automata cells. Hence, the CA-model is prepared to take into account mechanical influence of epithelium generating mechanical pressure to mesenchyme. It is shown that a tissue composed from living cells could be simulated by use of a tensegrity like structure which is composed from a set of nodes mutually interconnected by deformable bars. 


\section{References}

1. T. Toffoli and N. Margolus. Cellular Automata Theory. MIT Press, Cambridge, 1987.

2. A. Ilachinski. Cellular Automata: A Discrete Universe. World Scientific Publishing Co. Pte. Ltd., New Jersey, London, Singapore, Hong Kong, 2001.

3. S. Wolfram. A New Kind of Science. Wolfram Media Inc., Champaign, 2002.

4. D.E. Ingber. The architecture of life. SCIENTIFIC AMERICAN, 278(1):48-57, Jan 1998.

5. D.E. Ingber S.R. Heidemann P. Lamoreux and R.E. Buxbaum. Opposing views on tensegrity as a structural framework for understanding cell mechanics. J Appl Physiol, 89(4):1663-1670, Oct 2000.

6. P. Lamoreux S.R. Heidemann and R.E. Buxbaum. Opposing views on tensegrity as a structural framework for understanding cell mechanics. J Appl Physiol, 89:1670 1674, Oct 2000.

7. D.E. Ingber. Opposing views on tensegrity as a structural framework for understanding cell mechanics - rebuttals. J Appl Physiol, 89:1674-1677, Oct 2000.

8. D.E. Ingber S.R. Heidemann P. Lamoreux and R.E. Buxbaum. Opposing views on tensegrity as a structural framework for understanding cell mechanics - rebuttals. J Appl Physiol, 89:1677-1678, Oct 2000.

9. P. Hajela and B. Kim. On the use of energy minimization for ca based analysis in elasticity. Struct Multidisc Optim, 23:24-33, Dec 2001.

10. E. Kita and T. Toyoda. Structural design using cellular automata. Struct Multidisc Optim, 19:64-73, Mar 2000.

11. Z. Gurdal and B. Tatting. Cellular automata for design of truss structures with linear and nonlinear response. In 8th AIAA/USAF/NASA/ISSMO Symposium on Multidisciplinary Analysis and Optimization, pages 1-11, Long Beach, CA, Sep 2000. American Institute for Aeronautics and Astronautics.

12. Pilot F. and Lecuit T. Compartmentalized morphogenesis in epithelia: From cell to tissue shape. DEVELOPMENTAL DYNAMICS, 232(3):685-694, Mar 2005.

13. Hay E.D. The mesenchymal cell, its role in the embryo, and the remarkable signaling mechanisms that create it. DEVELOPMENTAL DYNAMICS, 233(3):706-720, Jul 2005.

14. Ball EMA and Risbridger GP. Activins as regulators of branching morphogenesis. DEVELOPMENTAL BIOLOGY, 238(1):1-12, Oct 2001.

15. S.R. Heidemann and D. Wirtz. Towards a regional approach to cell mechanics. TRENDS in Cell Biology, 14(4):160-166, Apr 2004. 